\title{
Thermodynamic Properties of the Superconducting State in the $\mathrm{K}_{3} \mathrm{C}_{60}$ Fulleride
}

\author{
R. SzCZĘ́SNIAK ${ }^{a, *}$ AND A. BARASIŃSKI ${ }^{b}$ \\ ${ }^{a}$ Institute of Physics, Częstochowa University of Technology, al. Armii Krajowej 19, 42-200 Częstochowa, Poland \\ ${ }^{b}$ Institute of Physics, University of Zielona Góra, Prof. Szafrana 4a, 65-516 Zielona Góra, Poland
}

(Received May 14, 2009; in final form November 10, 2009)

\begin{abstract}
In the framework of the Eliashberg formalism, we have calculated the thermodynamic properties of the superconducting state in $\mathrm{K}_{3} \mathrm{C}_{60}$ (the critical temperature $T_{\mathrm{C}}=19.50 \mathrm{~K}$ ). We have obtained the following results: (i) The critical value of the Coulomb pseudopotential is equal to 0.387. (ii) The values of the ratios $R_{1} \equiv 2 \Delta(0) / k_{\mathrm{B}} T_{\mathrm{C}}$ and $R_{2} \equiv \Delta C\left(T_{\mathrm{C}}\right) / C^{\mathrm{N}}\left(T_{\mathrm{C}}\right)$ are bigger than in the BCS model; $R_{1}=4.01$ and $R_{2}=1.58$. (iii) The electron effective mass $m_{\mathrm{e}}^{*}$ reaches the highest value for $T=T_{\mathrm{C}} ;\left[m_{\mathrm{e}}^{*}\right]_{\max }=2.86 m_{\mathrm{e}}$, where $m_{\mathrm{e}}$ is the bare electron mass. Additionally, we have given the analytical expressions for $T_{\mathrm{C}}, \Delta(T), R_{1}$ and $R_{2}$.
\end{abstract}

PACS numbers: $74.20 . \mathrm{Fg}, 74.70 . \mathrm{Wz}$

\section{Introduction}

The superconducting state in $\mathrm{K}_{3} \mathrm{C}_{60}$ was discovered in 1991 [1]. Since that time, the thermodynamic properties of this compound were very intensively studied. The brief discussion of the achieved results can be found in [2].

Despite the accumulation of the relatively large amount of the experimental data, none of the proposed models is still not fully accepted. The existing models of the superconducting state in $\mathrm{K}_{3} \mathrm{C}_{60}$ can be divided into two principal groups. The first one includes theories assuming that the superconducting state is induced by the electron-phonon interaction [3-6]. The second one contains theories that try to explain the formation of the superconducting state in the framework of the non-phonon pairing mechanism [7].

Below, we present the experimental data which clearly indicate that the superconducting phase in $\mathrm{K}_{3} \mathrm{C}_{60}$ is induced by the electron-phonon interaction.

First, the non-zero isotope effect was observed. In the case of the complete substitution of the carbon isotope ${ }^{12} \mathrm{C}$ by ${ }^{13} \mathrm{C}$ the isotope coefficient $(\alpha)$ is equal to $0.3 \pm 0.06$ [8]. On the other hand, the very high value of $\alpha$ equal to 1.43 can be achieved for the partial substitution [9]. It is worth to notice that the isotope effect is not created by the alkaline metals; it is evidenced by the results obtained for the fulleride $\mathrm{Rb}_{3} \mathrm{C}_{60}$ where the rubidium isotope ${ }^{85} \mathrm{Rb}$ has been replaced with the isotope ${ }^{87} \mathrm{Rb}[10]$. On the basis of the quoted facts one should suppose that the forming of the superconducting phase in $\mathrm{K}_{3} \mathrm{C}_{60}$ is connected with the existence of the coupling

* corresponding author; e-mail: szczesni@mim.pcz.czest.pl between electrons and the vibrations of the carbon atoms in $\mathrm{C}_{60}$ molecules.

Second, the order parameter has a pure $s$-wave symmetry [11-14]. This fact can be explained in a natural way by taking into consideration an assumption that the electron-phonon interaction is responsible for the superconducting phase induction. Let us notice that the pure electron pairing mechanism leads to different form of the wave symmetry; in particular, $d$-wave symmetry or mixed symmetry [15].

Some theoretical considerations also confirm our point of the view. The initial calculations conducted with the use of the Eliashberg equations indicate that in the case of $\mathrm{K}_{3} \mathrm{C}_{60}$ the electron-phonon interaction can independently induce the superconducting phase [16]. According to that, the precise calculation of the basic thermodynamic parameters seems to be an essential matter.

In the presented paper, we will determine the most relevant thermodynamic quantities that characterize the superconducting phase in $\mathrm{K}_{3} \mathrm{C}_{60}$. To achieve that, we will numerically solve the Eliashberg equations [17].

From the mathematical point of view it is a complicated problem because the Eliashberg equations form a set of the non-linear equations. Additionally, we will supplement the numerical analysis by the analytical approach. The obtained theoretical results will be also compared with the experimental data.

\section{The Eliashberg equations}

The set of the Eliashberg equations can be written in the following form [18]: 


$$
\begin{aligned}
& Z_{l} \Delta_{l}=\frac{\pi}{\beta} \sum_{m=1}^{M} \frac{\left[K^{+}(l, m)-2 \mu(m)\right]}{\sqrt{\omega_{m}^{2}+\Delta_{m}^{2}}} \Delta_{m}, \\
& Z_{l}=1+\frac{\pi}{\beta} \sum_{m=1}^{M} \frac{K^{-}(l, m)}{\sqrt{\omega_{m}^{2}+\Delta_{m}^{2}}} \frac{\omega_{m}}{\omega_{l}},
\end{aligned}
$$

where the symbol $\Delta_{l} \equiv \Delta\left(\mathrm{i} \omega_{l}\right)$ denotes the order parameter and $Z_{l} \equiv Z\left(\mathrm{i} \omega_{l}\right)$ is the wave function renormalization factor. The Matsubara frequencies are defined by the formula: $\omega_{l} \equiv(\pi / \beta)(2 l-1)$, where $\beta$ is the inverse temperature; $\beta \equiv\left(k_{\mathrm{B}} T\right)^{-1}$. In the paper we assume that the upper limit in the sum $(M)$ is equal to 800 .

The functions $K^{ \pm}(l, m)$ are given by the expression: $K^{ \pm}(l, m) \equiv K(l-m) \pm K(l+m-1)$, where $K(l-m)$ denotes the pairing kernel

$$
K(l-m) \equiv \int_{0}^{\Omega_{\max }} \mathrm{d} \Omega \frac{\alpha^{2} F(\Omega) 2 \Omega}{\left(\omega_{l}-\omega_{m}\right)^{2}+\Omega^{2}} .
$$

The Eliashberg function $\left(\alpha^{2} F(\Omega)\right)$ was determined in [16]; the value of the maximum phonon frequency $\left(\Omega_{\max }\right)$ is equal to $242 \mathrm{meV}$.

The symbol $\mu(m)$ denotes the function that models the influence of the Coulomb repulsion on the superconducting state

$$
\mu(m) \equiv \mu_{\mathrm{C}}^{*} \Theta\left(\omega_{\mathrm{c}}-\left|\omega_{m}\right|\right),
$$

where the critical value of the Coulomb pseudopotential $\left(\mu_{\mathrm{C}}^{*}\right)$ will be calculated in the next section. Symbol $\Theta$ stands for the Heaviside unit function; the phonon cut-off frequency $\left(\omega_{\mathrm{c}}\right)$ is equal to $1 \mathrm{eV}[16]$.

\section{The numerical and analytical results}

\subsection{The critical value of the Coulomb pseudopotential}

The Coulomb pseudopotential is defined by the expression: $\mu^{*} \equiv \rho(0) V /\left[1+\rho(0) V \ln \left(\omega_{\mathrm{P}} / \omega_{\mathrm{D}}\right)\right]$, where $\rho(0)$ is the electronic density of states at the Fermi level and $V$ is the Coulomb potential. The symbols $\omega_{\mathrm{P}}$ and $\omega_{\mathrm{D}}$ denote the electronic plasma frequency and the Debye phonon frequency, respectively [19]. We notice that the critical value of the Coulomb pseudopotential must be very precisely determined, otherwise it is impossible to compare the theoretical predictions with the experimental data quantitatively. The exact analysis of this issue is presented below.

In Fig. 1A we show the dependence of the order parameter $\Delta_{m}$ on the number $m$ for the selected values of $\mu^{*}$; calculations were made for $T=T_{\mathrm{C}}$. Results presented in Fig. 1A prove that for $\mu^{*}<\mu_{\mathrm{C}}^{*}$ the order parameter takes the highest value always for $m=1$. Thus, the critical value of the Coulomb pseudopotential can be determined by using the condition

$$
\left[\Delta_{m=1}\left(\mu_{\mathrm{C}}^{*}\right)=0\right]_{T=T_{\mathrm{C}}} .
$$

The full dependence of $\Delta_{m=1}$ on $\mu^{*}$ is shown in Fig. 1B. On the basis of the achieved results we have obtained that the critical value of the Coulomb pseudopotential is equal to 0.387 . In comparison with other classical superconductors the value of $\mu_{\mathrm{C}}^{*}$ for $\mathrm{K}_{3} \mathrm{C}_{60}$ is very high [20].

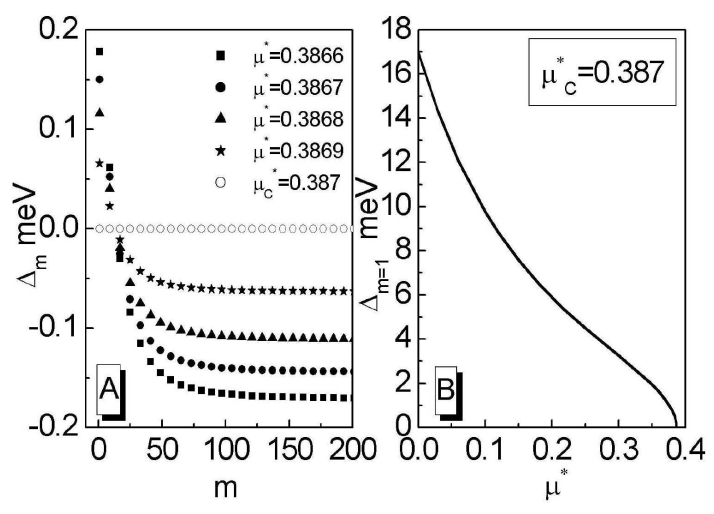

Fig. 1. (A) The order parameter as a function of the number $m$ for selected values of the Coulomb pseudopotential. In the figure first 200 values of $\Delta_{m}$ are presented. (B) The full dependence of $\Delta_{m=1}$ on the Coulomb pseudopotential.

\subsection{The formula for the critical temperature}

For the classical low-temperature superconductors, the value of the critical temperature is determined by a good approximation on the basis of the Allen-Dynes (AD) formula [21]:

$$
k_{\mathrm{B}} T_{\mathrm{C}}=f_{1} f_{2} \frac{\omega_{\mathrm{ln}}}{1.2} \exp \left(\frac{-1.04(1+\lambda)}{\lambda-\mu^{*}(1+0.62 \lambda)}\right),
$$

where the parameters and functions included in the expression (6) are determined in Table.

TABLE

The definitions of quantities in Eq. (6). The parameters $\lambda$ and $\omega_{1 n}$ are called the electron-phonon coupling constant and the logarithmic phonon frequency, respectively; $\omega_{2}$ is the second moment of the normalized weight function: $g(\Omega) \equiv \frac{2}{\lambda \Omega} \alpha^{2} F(\Omega)$. The symbols $f_{1}$ and $f_{2}$ denote the strong-coupling correction function and the shape correction function, respectively.

\begin{tabular}{cc}
\hline \hline Parameter or function & Value \\
\hline$\lambda \equiv 2 \int_{0}^{\Omega_{\max }} \mathrm{d} \Omega \frac{\alpha^{2} F(\Omega)}{\Omega}$ & 1.217 \\
$\omega_{\ln } \equiv \exp \left(\frac{2}{\lambda} \int_{0}^{\Omega_{\max }} \mathrm{d} \Omega \frac{\alpha^{2} F(\Omega)}{\Omega} \ln (\Omega)\right)$ & $35.66 \mathrm{meV}$ \\
$\omega_{2} \equiv \frac{2}{\lambda} \int_{0}^{\Omega_{\max }} \mathrm{d} \Omega \alpha^{2} F(\Omega) \Omega$ & $9.4 \mathrm{eV}$ \\
$f_{1} \equiv\left[1+\left(\frac{\lambda}{\Lambda_{1}}\right)^{\frac{3}{2}}\right]^{\frac{1}{3}}$ & \\
$f_{2} \equiv 1+\frac{\left(\frac{\sqrt{\omega_{2}}}{\omega_{\ln }}-1\right) \lambda^{2}}{\lambda^{2}+\Lambda_{2}^{2}}$ &
\end{tabular}

In the case of $\mathrm{K}_{3} \mathrm{C}_{60}$ the $\mathrm{AD}$ formula does not work. Particularly, for $\mu_{\mathrm{C}}^{*}=0.387$ we obtain $\left[T_{\mathrm{C}}\right]_{\mathrm{AD}}=4.9 \mathrm{~K}$ (see also Fig. 2). 


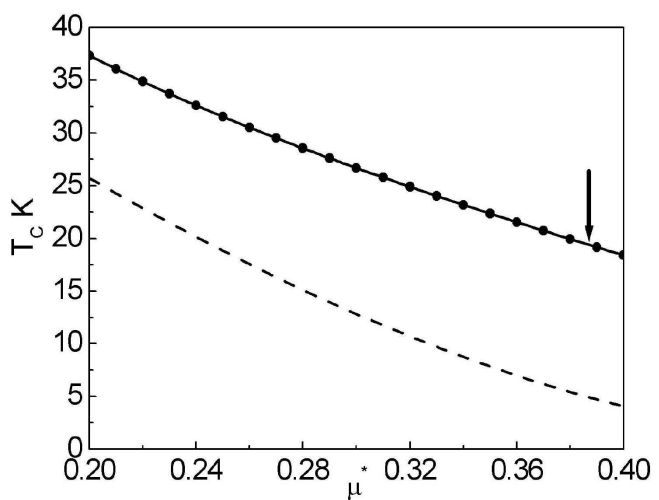

Fig. 2. The critical temperature as a function of the Coulomb pseudopotential. Dashed line represents the calculation of $T_{\mathrm{C}}$ using the classical Allen-Dynes formula. The black circles correspond to the exact numerical solutions of the Eliashberg equations. Solid line represents the calculation of $T_{\mathrm{C}}$ using our analytical scheme $((7)$ and $(8))$. The black arrow denotes the experimental value of the critical temperature for $\mathrm{K}_{3} \mathrm{C}_{60}$.

In the paper, we have modified the classical AllenDynes formula by choosing again the numerical coefficients in $\Lambda_{1}$ and $\Lambda_{2}$. We have used the least-squares analysis and 250 numerical solutions of the Eliashberg equations for selected values of $\mu^{*}$. The result has the form

$$
\begin{aligned}
& \Lambda_{1} \equiv 2\left(1+0.05 \mu^{*}\right), \\
& \Lambda_{2} \equiv-0.05\left(1-166 \mu^{*}\right)\left(\frac{\sqrt{\omega_{2}}}{\omega_{\ln }}\right) .
\end{aligned}
$$

The dependence of the critical temperature on the Coulomb pseudopotential obtained by using the exact numerical analysis and in the framework of our analytical scheme is shown in Fig. 2. It is easy to notice that the agreement is excellent.

\subsection{The order parameter function}

In the Eliashberg formalism, in order to reconstruct the experimental dependence of the order parameter on the temperature, the form of the function $\Delta_{m}$ for selected values of the temperature should be determined in the first step.

In Fig. 3A we show the order parameter function on the imaginary axis. We select the following range of the temperature: $T \in(4.64,19.50) \mathrm{K}$. We notice that for $T<4.64 \mathrm{~K}$ the variation of the order parameter curve is negligible. This fact is presented in Fig. 3B.

In the next step, the order parameter function on the real axis should be calculated. It can be done with the use of the analytical continuation method described in [22]. In the framework of this approach, the order parameter function is determined as the ratio of two polynomials

$$
\Delta(\omega)=\frac{p_{\Delta 1}+p_{\Delta 2} \omega+\ldots+p_{\Delta r} \omega^{r-1}}{q_{\Delta 1}+q_{\Delta 2} \omega+\ldots+q_{\Delta r} \omega^{r-1}+\omega^{r}},
$$

where $p_{\Delta n}$ and $q_{\Delta n}$ are the numerical coefficients. In
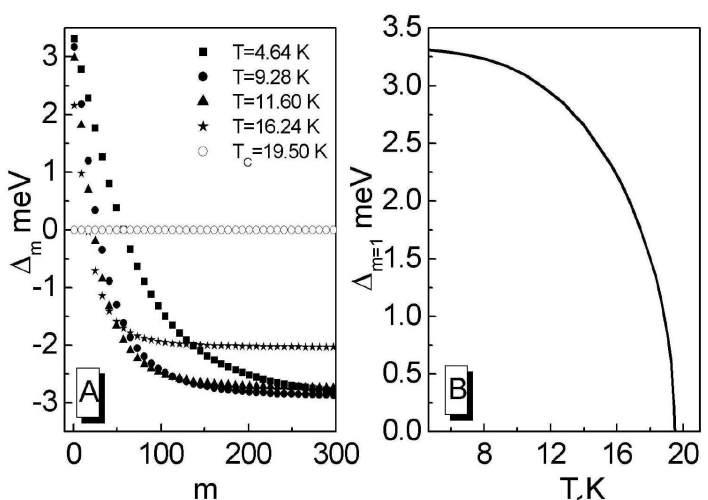

Fig. 3. (A) The dependence of the order parameter on the number $m$ for selected temperatures. In the figure first 300 values of $\Delta_{m}$ are presented. (B) The value of the order parameter for $m=1$ as a function of the temperature.

our case, the maximum value of the exponent $r$ is equal to 400 .

As an example, in Fig. 4 we show the real (Re) and imaginary $(\mathrm{Im})$ part of the order parameter on the real axis, calculated for $T=4.64 \mathrm{~K}$.

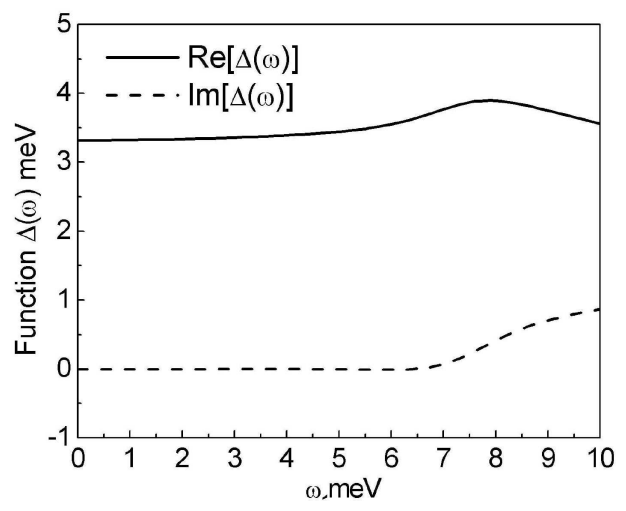

Fig. 4. The dependence of the real and imaginary part of the order parameter on the frequency for $T=4.64 \mathrm{~K}$.

Finally, the value of the order parameter for the given temperature is determined on the basis of the equation

$$
\Delta(T)=\operatorname{Re}[\Delta(\omega=\Delta(T), T)] .
$$

In the case of $\mathrm{K}_{3} \mathrm{C}_{60}$, the full dependence of the order parameter on the temperature can be described by the following expression:

$$
\Delta(T)=\Delta\left(T_{1}\right) \sqrt{1-\left(\frac{T-T_{1}}{T_{\mathrm{C}}-T_{1}}\right)^{\beta}},
$$

where $T_{1}=4.64 \mathrm{~K}, \Delta\left(T_{1}\right)=3.37 \mathrm{meV}$ and $\beta=1.94$.

On the basis of achieved results, we can calculate the ratio of the order parameter near the zero temperature $(\Delta(0))$ to critical temperature: $R_{1} \equiv 2 \Delta(0) / k_{\mathrm{B}} T_{\mathrm{C}}$. In particular, we assume that $\Delta(0) \simeq \Delta\left(T_{1}\right)$. The following evaluation is obtained: $R_{1}=4.01$. The above result 
means that the value of $R_{1}$ is greater than the value predicted by the BCS theory, where $\left[R_{1}\right]_{\mathrm{BCS}}=3.53[23]$.

Additionally, we compare the theoretical and experimental values of $R_{1}$. We find that our result is in good agreement with the NMR data, where $R_{1}=4.3$ [24]. However, the optical and tunneling methods give the values that differ from our prediction $[2,13]$.

The numerical analysis of the Eliashberg equations is the complicated problem, therefore we have derived the analytical expression for $R_{1}$ :

$$
\frac{R_{1}}{\left[R_{1}\right]_{\mathrm{BCS}}}=1+138\left(p_{a} \frac{k_{\mathrm{B}} T_{\mathrm{C}}}{\omega_{\mathrm{ln}}}\right)^{2} \ln \left(\frac{1}{p_{a}} \frac{\omega_{\mathrm{ln}}}{2 k_{\mathrm{B}} T_{\mathrm{C}}}\right),
$$

where the correction $p_{a}$ has the form: $p_{a} \equiv f_{1} / 3 f_{2}$. In obtaining Eq. (12) the spirit of the Mitrović-ZarateCarbotte formula was followed [20]. We notice that the coefficients 138, 2 and 3 were chosen to fit to the numerical data for 65 selected values of the Coulomb pseudopotential.

\subsection{The free energy and the specific heat}

We calculate the free energy difference between the superconducting and normal state $(\Delta F)$ on the basis of formula originally brought out by Bardeen and Stephen [25]:

$$
\begin{aligned}
& \frac{\Delta F}{\rho(0)}=-\frac{2 \pi}{\beta} \sum_{m=1}^{M}\left(\sqrt{\omega_{m}^{2}+\Delta_{m}^{2}}-\left|\omega_{m}\right|\right) \\
& \quad \times\left(Z_{m}^{\mathrm{S}}-Z_{m}^{\mathrm{N}} \frac{\left|\omega_{m}\right|}{\sqrt{\omega_{m}^{2}+\Delta_{m}^{2}}}\right),
\end{aligned}
$$

where the superscripts $\mathrm{S}$ and $\mathrm{N}$ denote the superconducting and normal state, respectively.

The dependence of the free energy difference on the temperature in the range from $4.64 \mathrm{~K}$ to $19.50 \mathrm{~K}$ is plotted in Fig. 5A. As it is easy to note, the function $\Delta F$ is increasing with the growth of the temperature, reaching its maximum equal to zero at $T=T_{\mathrm{C}}$. Negative values of $\Delta F$ prove explicitly that below $T_{\mathrm{C}}$ the superconducting phase is thermodynamically stable.
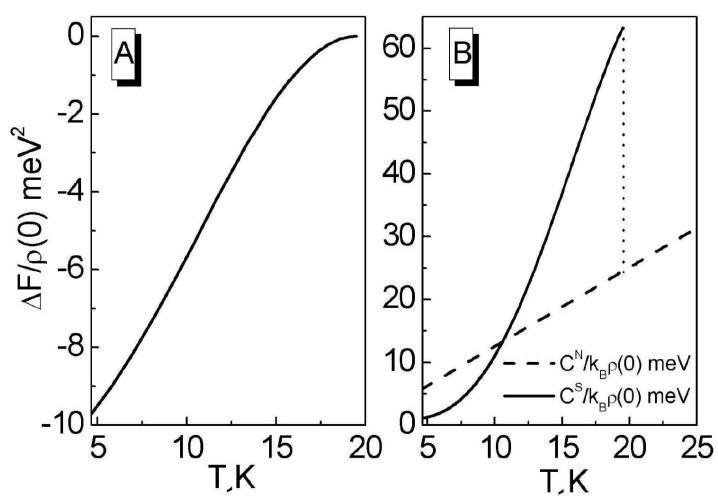

Fig. 5. (A) The free energy difference between the superconducting and normal state as a function of the temperature. (B) The specific heat in the normal and superconducting state as a function of the temperature.
On the basis of the function $\Delta F$ we calculate the specific heat difference between the superconducting and normal state $\left(\Delta C \equiv C^{\mathrm{S}}-C^{\mathrm{N}}\right)$ :

$$
\frac{\Delta C(T)}{k_{\mathrm{B}} \rho(0)}=-\frac{1}{\beta} \frac{\mathrm{d}^{2}[\Delta F / \rho(0)]}{\mathrm{d}\left(k_{\mathrm{B}} T\right)^{2}} .
$$

On the other hand, the normal state specific heat can be determined by using the expression

$$
\frac{C^{\mathrm{N}}(T)}{k_{\mathrm{B}} \rho(0)}=\frac{\gamma}{\beta},
$$

where $\gamma \equiv \frac{2}{3} \pi^{2}(1+\lambda)$.

In Fig. 5B we show the dependence of $C^{\mathrm{N}}$ and $C^{\mathrm{S}}$ on the temperature. The specific heat jump at $T_{\mathrm{C}}$ can be easily seen.

Below, we calculate the dimensionless ratio $R_{2} \equiv$ $\Delta C\left(T_{\mathrm{C}}\right) / C^{\mathrm{N}}\left(T_{\mathrm{C}}\right)$. In the framework of the BCS theory the parameter $R_{2}$ is the universal constant of the model and $\left[R_{2}\right]_{\mathrm{BCS}}=1.43[23]$. As a result of the conducted analysis we have obtained bigger value than in BCS theory: $R_{2}=1.58$. We notice that our value of $R_{2}$ agree with the experimental data in the limit of the measure error; $\left[R_{2}\right]_{\text {expt }}=2.0 \pm 0.5[26]$.

Finally, the value of the ratio $R_{2}$ can be also calculated analytically by using the formula

$$
\frac{R_{2}}{\left[R_{2}\right]_{\mathrm{BCS}}}=1+195\left(p_{b} \frac{k_{\mathrm{B}} T_{\mathrm{C}}}{\omega_{\mathrm{ln}}}\right)^{2} \ln \left(\frac{1}{p_{b}} \frac{\omega_{\mathrm{ln}}}{3 k_{\mathrm{B}} T_{\mathrm{C}}}\right),
$$

where $p_{b} \equiv f_{1} / 4 f_{2}$. We have chosen the coefficients 195 , 3 , and 4 from fits to the numerical data for 50 selected values of $\mu^{*}$. We have derived the expression (16) in the scheme proposed by Marsiglio and Carbotte [27].

\subsection{The electron effective mass}

The electron-phonon coupling leads to the increase of the electron effective mass $\left(m_{\mathrm{e}}^{*}\right)$. In the framework of the Eliashberg formalism, the ratio of the electron effective mass to the bare electron mass $\left(m_{\mathrm{e}}\right)$ is given, within a good approximation, by the value of the wave function renormalization factor for $m=1$.

In Fig. 6 we show the dependence of the wave function renormalization factor on the number $m$ for selected values of the temperature. It can be noticed that for $m=1$ there is always a correspondence of the maximum value of the wave function renormalization factor. The inset in Fig. 6 presents a full dependence of $Z_{m=1}$ on the temperature. On the basis of the plotted curve we claim that the effective mass takes the highest value for $T=T_{\mathrm{C}}$.

Below we calculate precisely the electron effective mass for $T=T_{\mathrm{C}}$. To achieve that, the expression $m_{\mathrm{e}}^{*} / m_{\mathrm{e}}=$ $Z(0)$ was used, where $Z(0)$ is the value of the wave function renormalization factor on the real axis for $\omega=0$. The function $Z(\omega)$ can be determined on the basis of the analytical continuation theorem [22]. In our case

$$
Z(\omega)=\frac{p_{Z 1}+p_{Z 2} \omega+\ldots+p_{Z r} \omega^{r-1}}{q_{Z 1}+q_{Z 2} \omega+\ldots+q_{Z r} \omega^{r-1}+\omega^{r}},
$$

where $p_{Z n}$ and $q_{Z n}$ are the number coefficients, $r$ is equal to 400 . 


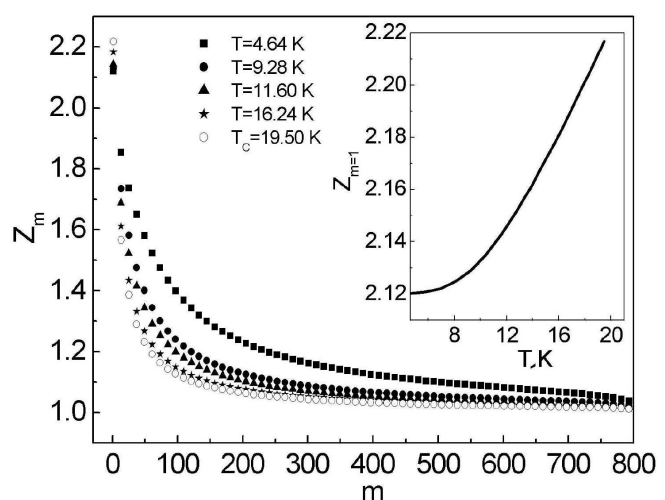

Fig. 6. The dependence of the wave function renormalization factor on the number $m$ for selected values of the temperature. In the inset there is a maximum value of the wave function renormalization factor as a function of the temperature.

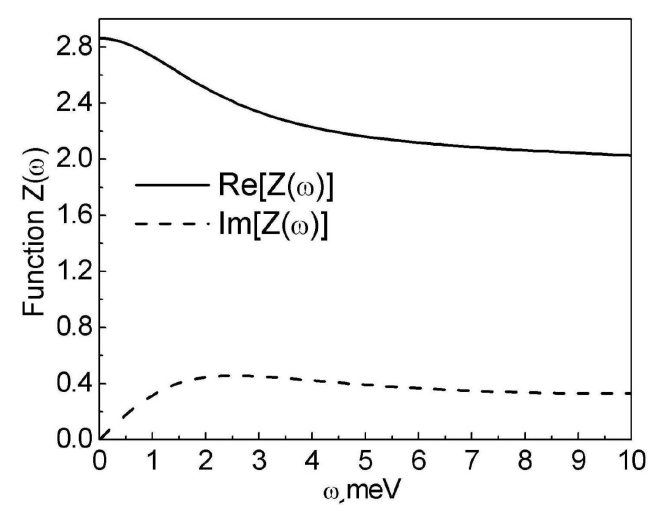

Fig. 7. The real and imaginary part of the wave function renormalization factor on the real axis.

In Fig. 7 we show the dependence of the real and imaginary part of the wave function renormalization factor on the frequency. For $\omega=0$ the non-zero value is taken only by a real part: $\operatorname{Re}[Z(0)]=2.862$. The above result proves that in $\mathrm{K}_{3} \mathrm{C}_{60}$ the electron-phonon interaction strongly renormalizes the electron effective mass.

\section{Concluding remarks}

In the paper, we have determined the thermodynamic properties of the superconducting state in the $\mathrm{K}_{3} \mathrm{C}_{60}$.

In the first step, we have shown that the critical value of the Coulomb pseudopotential is equal to 0.387 . It is a high value in comparison with the value of $\mu_{\mathrm{C}}^{*}$ calculated for other classical superconductors [20].

In the second step, we have given the modified AllenDynes formula which reproduces very well the experimental value of the critical temperature. We have determined also the dependence of the order parameter on the temperature. The obtained results enabled the calculation of the ratio $R_{1}$ whose value equals 4.01 . At this moment it is difficult to state whether the theoretical value of $R_{1}$ agrees with the experimental value because the experimental results given in the literature significantly differ.

Next, we have calculated the dependence of the free energy difference on the temperature. By using the function $\Delta F$ we have estimated the specific heat-jump at $T_{\mathrm{C}}$ and the value of the ratio $R_{2}$ (1.58). We have shown that the theoretical value of $R_{2}$ agrees with the experimental result in the limit of the measure error.

In the last step, we have calculated the electron effective mass. We have shown that the effective mass reaches the highest value for $T=T_{\mathrm{C}}$. The following estimation was obtained: $\left[m_{\mathrm{e}}^{*}\right]_{\max }=2.862 m_{\mathrm{e}}$. The above result proves that the electron-phonon interaction strongly renormalizes the electron effective mass.

In the future, we will answer the question whether in the case of $\mathrm{K}_{3} \mathrm{C}_{60}$ the vertex corrections for electronphonon interaction have a really relevant meaning [28].

\section{Acknowledgments}

The authors wish to thank: (i) Prof. F. Marsiglio for the disclosure of the Eliashberg function for $\mathrm{K}_{3} \mathrm{C}_{60}$. (ii) Prof. K. Dziliński for the creating excellent working conditions and the financial support. Some computational resources have been provided by the RSC Computing Center.

\section{References}

[1] (a) A.F. Hebard, M.J. Rosseinsky, R.C. Haddon, D.W. Murphy, S.H. Glarum, T.T.M. Palstra, A.P. Ramirez, A.R. Kortan, Nature 350, 600 (1991); (b) K. Holczer, O. Klein, S.M. Huang, S.B. Kaner, K.J. Fu, R.L. Whetten, F. Diederich, Science 252, 1154 (1991).

[2] O. Gunnarsson, Rev. Mod. Phys. 69, 575 (1997).

[3] C.M. Varma, J. Zaanen, K. Raghavachari, Science 254, 989 (1991).

[4] M.A. Schlütter, M. Lannoo, M. Needels, G.A. Baraff, D. Tomanek, Phys. Rev. Lett. 68, 526 (1992).

[5] R. Dagani, Chem. Eng. News 20, 4 (1994).

[6] K. Prassides, H.W. Kroto, R. Taylor, D.R. Waltron, V.I.F. David, J. Tomkinson, R.C. Haddon, M.J. Rosseinsky, D.W. Murphy, Carbon 30, 1277 (1992).

[7] (a) R.A. Iishi, M.S. Dresselhaus, Phys. Rev. B 45, 2597 (1992); (b) S. Chakrawarty, S.A. Kivelson, M.J. Salkola, S. Tewari, Science 256, 1306 (1992).

[8] C.-C. Chen, C.L. Lieber, J. Am. Chem. Soc. 114, 3141 (1992).

[9] A.A. Zakhidov, K. Imaeda, D.M. Petty, K. Yakushi, H. Inokuchi, K. Kikuchi, I. Ikemoto, S. Suzuki, Y. Achiba, Phys. Lett. A 164, 355 (1992).

[10] T.W. Ebbesen, J.S. Tsai, K. Tanigaki, H. Hiura, Y. Shimakawa, Y. Kubo, I. Hirosawa, J. Mizuki, Physica C 203, 163 (1992).

[11] Z. Zhang, C. Chen, C.M. Lieber, Science 254, 1619 (1991).

[12] D. Koller, M.C. Martin, L. Mihály, G. Mihály, G. Oszányi, G. Baumgartner, L. Forró, Phys. Rev. Lett. 77, 4082 (1996). 
[13] L. Degiorgi, G. Briceno, M.S. Fuhrer, A. Zettl, P. Wachter, Nature 369, 541 (1994).

[14] L. Degiorgi, E.J. Nicol, O. Klein, G. Grüner, P. Wachter, S.-M. Huang, J. Wiley, R.B. Kaner, Phys. Rev. B 49, 7012 (1994).

[15] (a) M. Mierzejewski, J. Zieliński, Phys. Rev. B 56, 1 (1997); (b) H.J.H. Smilde, A.A. Golubov, Ariando, G. Rijnders, J.M. Dekkers, S. Harkema, D.H.A. Blank, H. Rogalla, H. Hilgenkamp, Phys. Rev. Lett. 95, 257001 (2005).

[16] F. Marsiglio, T. Startseva, J.P. Carbotte, Phys. Lett. A 245, 172 (1998).

[17] For discussion of the Eliashberg equations [originally formulated by G.M. Eliashberg, Sov. Phys. - JETP 11, 696 (1960)] we refer to: (a) P.B. Allen, B. Mitrović, in: Solid State Physics: Advances in Research and Applications, Vol. 37, Eds. H. Ehrenreich, F. Seitz, D. Turnbull, Academic, New York 1982, p. 1; (b) J.P. Carbotte, F. Marsiglio, in: The Physics of Superconductors, Vol. 1, Eds. K.H. Bennemann, J.B. Ketterson, Springer, Berlin 2003, p. 223.

[18] R. Szczęśniak, Phys. Status Solidi B 244, 2538 (2007); R. Szczęśniak, Acta Phys. Pol. A 109, 179 (2006).
[19] W.L. McMillan, Phys. Rev. 167, 331 (1968).

[20] B. Mitrović, H.G. Zarate, J.P. Carbotte, Phys. Rev. B 29, 184 (1984).

[21] P.B. Allen, R.C. Dynes, Phys. Rev. B 12, 905 (1975).

[22] K.S.D. Beach, R.J. Gooding, F. Marsiglio, Phys. Rev. B 61, 5147 (2000).

[23] (a) J. Bardeen, L.N. Cooper, J.R. Schrieffer, Phys. Rev. 106, 162 (1957); (b) J. Bardeen, L.N. Cooper, J.R. Schrieffer, Phys. Rev. 108, 1175 (1957).

[24] S. Sasaki, A. Matsuda, C.W. Chu, J. Phys. Soc. Japan 63, 1670 (1994).

[25] J. Bardeen, M. Stephen, Phys. Rev. 136, A1485 (1964).

[26] I.M. Tang, P. Winotai, Phys. Lett. A 208, 339 (1995).

[27] F. Marsiglio, J.P. Carbotte, Phys. Rev. B 33, 6141 (1986).

[28] (a) L. Pietronero, S. Strässler, Europhys. Lett. 18, 627 (1992); (b) L. Pietronero, S. Strässler, C. Grimaldi, Phys. Rev. B 52, 10516 (1995). 